\title{
ASPECTOS DA AIDS DE INTERESSE PARA O OFTALMOLOGISTA
}

\author{
SOME ASPECTS OF AIDS WITH INTEREST TO OPHTHALMOLOGISTS \\ José Fernando de Castro Figueiredo ${ }^{1}$; Maria de Lourdes Veronese Rodrigues ${ }^{2}$; Nivaldo Vieira de Souza ${ }^{2}$; \\ Erasmo Romão ${ }^{2} \&$ Fernanda Teixeira da Silva Krieger ${ }^{2}$
}

\begin{abstract}
'Docente da Divisão de Moléstias Infecciosas e Tropicais do Departamento de Clínica Médica; ${ }^{2}$ Docente do Departamento de Oftalmologia e Otorrinolaringologia. Faculdade de Medicina de Ribeirão Preto da Universidade de São Paulo.

CorResPondênCIA: José Fernando de Castro Figueiredo - Divisão de Moléstias Infecciosas e Tropicais do Departamento de Clínica Médica, Faculdade de Medicina de Ribeirão Preto-USP, Avenida Bandeirantes, 3900, CEP: 14049-900, Ribeirão Preto, São Paulo, Brasil.
\end{abstract}

FIGUEIREDO JFC et al. Aspectos da aids de interesse para o oftalmologista. Medicina, Ribeirão Preto, 31: 577-583, out./dez. 1998.

RESUMO: Diversas especialidades médicas, em maior ou menor grau, estão se deparando com a aids, envolvidas nos planos diagnósticos, terapêuticos, profiláticos e de investigação. O oftalmologista tem papel de destaque no diagnóstico e na orientação terapêutica de algumas condições clínicas da aids. As complicações oculares mais comuns nos pacientes com aids decorrem, quase sempre, da exacerbação de processos infecciosos latentes, adquiridos em época anterior à imunodepressão. Essas infecções tendem a se disseminar, causando manifestações sistêmicas variadas, com quadros clínicos nem sempre característicos. O acometimento ocular é mera manifestação local da disseminação do processo e a avaliação oftalmológica auxilia no estabelecimento do diagnóstico, uma vez que as alterações verificadas no exame fundoscópico podem ser indicativas da etiologia do processo. Nesse sentido, é feita uma revisão de alguns aspectos da aids que podem ser do interesse do oftalmologista.

UNITERMOS: Oftalmologia. Manifestações Oculares. Olho. Síndrome de Imunodeficiência Adquirida.

\section{INTRODUÇÃO}

\subsection{Considerações Gerais}

Com o aumento do número de casos de aids, verificado nos últimos anos, e levando-se em conta o freqüente acometimento do globo ocular nessa doença, tem sido comum o oftalmologista se deparar com tal problema numa primeira instância. Nessa condição, caberá a esse profissional suspeitar do diagnóstico de aids e fazer as orientações pertinentes, tais como solicitar os exames confirmatórios, comunicar o resultado ao paciente e encaminhá-lo para seguimento especializado. Além disso, o oftalmologista desempe- nha um papel importante para a avaliação e orientação diagnóstica e terapêutica das complicações oculares dos pacientes seguidos por outros profissionais, como parte da equipe multiprofissional que cuida desses pacientes. Participa ainda na elaboração e no desenvolvimento de pesquisas, envolvendo os diversos aspectos do acometimento ocular da aids (estudos epidemiológicos, ensaios clínicos, diagnóstico, tratamento e prevenção).

Neste contexto, seria importante uma revisão geral sobre a aids, objetivando uma sistematização de conceitos básicos de interesse para o oftalmologista. 


\subsection{Aspectos Epidemiológicos}

A Síndrome da Imunodeficiência Adquirida (aids) foi descrita em 1981, a partir da constatação de casos de sarcoma de Kaposi e pneumonia pelo Pneumocystis carinii em homossexuais masculi$\operatorname{nos}^{(1,2)}$ e atinge hoje proporções pandêmicas. Do início da epidemia até o final da década de noventa (90), estima-se que mais de trinta (30) milhões de pessoas no mundo tenham se infectado com o vírus da imunodeficiência humana (HIV), dos quais a maioria é de residentes no Continente Africano e no sul e sudeste da Ásia( ${ }^{(3)}$. No final de 1997 estimava-se que havia um milhão e trezentas mil (1.300.000) pessoas infectadas pelo HIV ou com aids na América Latina e no Caribe ${ }^{(3)}$ e, no Brasil, aproximadamente, cento e trinta e cinco mil (135.000) casos de aids em meados de $1998^{(4)}$.

A epidemia de aids no Brasil tem apresentado mudanças em algumas de suas características epidemiológicas, nos últimos anos. Na década de oitenta (80), havia predomínio absoluto do acometimento de pessoas do sexo masculino, com marcada transmissão através de relacionamento sexual homo/bissexual. Nos últimos anos, tem se verificado um aumento na incidência em mulheres (com o conseqüente aumento na transmissão vertical), aumento da transmissão através do uso de drogas ilícitas injetáveis e aumento da transmissão através do relacionamento heterossexual. Tem sido verificada, ainda, a expansão da epidemia dos grandes centros urbanos para as pequenas cidades e para o meio rural (interiorização), maior incidência em pessoas de classes sócio-econômicas mais baixas (proletarização) e maior acometimento dos adolescentes ${ }^{(4)}$.

\subsection{Aspectos Clínicos Gerais}

A evolução clínica de um paciente infectado pelo HIV é bastante variável, dependendo de fatores relacionados ao HIV e ao hospedeiro. Assim, podemos reconhecer três padrões dominantes de evolução: 8090\% dos pacientes evoluem de forma típica, com sobrevida média de aproximadamente dez (10) $\operatorname{anos}^{(5)}$; 5 a $10 \%$ evoluem para a aids de uma forma rápida, usualmente em três (3) a quatro (4) anos ${ }^{(6)}$; e cerca de 5\% dos indivíduos infectados permanecem assintomáticos por longos períodos de tempo, constituindo um grupo conhecido como "long-term survivors"(7).

A história natural da infecção pelo HIV pode ser dividida em três fases distintas: infecção aguda, período assintomático e período de doença (aids pro- priamente dita). A doença aguda passa despercebida na maioria dos infectados e o diagnóstico é feito somente em uma pequena parcela dos indivíduos. Quando sintomática, a doença aguda se manifesta através de um quadro clínico inespecífico, semelhante ao verificado na mononucleose infecciosa, e que se inicia cerca de três (3) a seis (6) semanas após o contágio. Nessa fase os pacientes podem apresentar febre, irritação na garganta, indisposição geral, mialgia, artralgia, linfoadenomegalia, exantemas maculopapulares, fotofobia e , raramente, meningoencefalite. Nessa etapa, usualmente, ainda não se encontram anticorpos específicos em circulação e o diagnóstico pode ser feito pela determinação da viremia, através da detecção de antígenos do HIV ou de seu genoma. A fase aguda é autolimitada, evoluindo para a cura em períodos variáveis de tempo (até doze (12) semanas após o início dos sintomas). Por essa época, os exames para a detecção de anticorpos anti-HIV já são positivos, na maioria dos $\operatorname{casos}^{(8)}$. Segue-se uma fase de latência clínica, de duração variável, como anteriormente relatado. Durante esse período, continua ocorrendo replicação viral no tecido linfóide, que resulta em sua destruição progressiva e em grave comprometimento da função imunológica, culminando com o aparecimento das complicações clínicas, indicativas da aids. Estas, se analisadas em conjunto com o número de linfócitos $\mathrm{CD}_{4}{ }^{+}$na circulação, podem permitir a classificação dos indivíduos infectados pelo HIV, conforme ilustrado abaixo ${ }^{(9)}$.

\begin{tabular}{|cccc|}
\hline \multicolumn{3}{|c|}{ Tabela I - Classificação da infecção pelo HIV em } \\
adolescentes e adultos \\
\hline Linfócitos $\mathrm{CD}_{4}{ }^{+}\left(\mathrm{mm}^{3}\right)$ & \multicolumn{4}{|c|}{ Categorias Clínicas* } \\
& A & B & C \\
\hline$>500$ & A1 & B1 & C1 \\
\hline $200-499$ & A2 & B2 & C2 \\
\hline$<200$ & A3 & B3 & C3 \\
\hline
\end{tabular}

* As categorias clínicas expressas na Tabela 1 podem ser definidas da seguinte maneira:

- Categoria A - consiste de uma ou mais das seguintes condições: infecção assintomática pelo HIV; linfoadenomegalia generalizada e persistente; infecção aguda primária pelo HIV (desde que não tenham ocorrido condições listadas nas categorias B ou C).

- Categoria $B$ - são condições que ocorrem em um paciente infectado pelo HIV e que preencham, pelo menos, um 
dos seguintes critérios: a) a condição é atribuída à infecção pelo HIV ou é indicativa de defeito de imunidade celular; ou b) a condição tem uma evolução clínica que é influenciada pela infecção pelo HIV. São exemplos de condições classificáveis como categoria B: angiomatose bacilar, candidíase de orofaringe, candidíase vulvovaginal, displasia cervical ou carcinoma "in situ" do cérvix uterino, sintomas constitucionais como febre ou diarréia, leucoplasia pilosa oral, Herpes zoster, acometendo mais de um dermátomo ou Herpes zoster recorrente, púrpura trombocitopênica idiopática, listeriose, doença pélvica inflamatória e neuropatia periférica.

- Categoria C - inclui as condições listadas como "caso indicativo de aids", a seguir relacionadas: candidíase de brônquios, traquéia ou pulmões, candidíase de esôfago, câncer cervical invasivo, criptococose extrapulmonar, criptosporidiose crônica intestinal, retinite pelo citomegalovírus, encefalopatia relacionada ao HIV, infecção crônica pelo Herpes simples, histoplasmose disseminada ou extrapulmonar, isosporíase crônica intestinal, sarcoma de Kaposi, linfoma de Burkitt, linfoma imunoblástico, linfoma primário do sistema nervoso central, infecção disseminada ou extrapulmonar pelo complexo Mycobacterium avium ou Mycobacterium kansasii, infecção pelo Mycobacterium tuberculosis, infecção disseminada ou extrapulmonar por outras espécies de Mycobacterium, pneumonia pelo Pneumocystis carinii, pneumonias de repetição, leucoencefalopatia multifocal progressiva, septicemia por Salmonella, toxoplasmose cerebral e síndrome consuntiva, devida ao HIV.

Pela classificação acima, são considerados como portadores de aids os indivíduos incluídos nas categorias A3, B3, C1, C2 e C3.

Deve ser ressaltado que muitas das complicações infecciosas do paciente com aids são decorrentes de processos infecciosos latentes, adquiridos em etapa anterior à imunodepressão; assim, podem apresentar peculiaridades regionais, de acordo com a prevalência das diferentes doenças. Estudos realizados no Brasil relatam a importância da infecção pelo bacilo da tuberculose, da reativação da doença de Chagas, da paracoccidioidomicose e da leishmaniose visceral, além da neurocriptococose, da neurotoxoplasmose, da pneumonia pelo Pneumocystis carinii, das pneumonias bacterianas e das candidíases ${ }^{(10,11,12)}$.

\section{MANIFESTAÇÕES OCULARES}

Desde as primeiras descrições da aids, tornou-se evidente a importância do acometimento ocular nessa doença. Além de muito freqüente, pode ser a primeira manifestação da síndrome ${ }^{(13)}$. Tais manifestações costumam ocorrer em fases mais avançadas da infecção pelo HIV, quando a contagem de linfócitos $\mathrm{CD}_{4}{ }^{+}$atinge valores abaixo de 100 células $/ \mathrm{mm}^{(3,14)}$.

As alterações oculares podem acometer os segmentos anterior e posterior, ou os anexos oculares. $\mathrm{O}$ segmento posterior é o mais comumente afetado e onde os danos funcionais têm repercussões mais graves. As manifestações oculares da aids podem ser divididas em cinco (5) categorias $^{(15)}$ : I - alterações da microvasculatura; II - infecções oportunistas; III neoplasias; IV- distúrbios neurooftalmológicos e; Voutras alterações.

Estudos realizados no Brasil têm revelado, com pequenas variações regionais, que a retinite pelo citomegalovírus (CMV) é a infecção mais comum ${ }^{(16,17)}$. Um estudo das manifestações oculares da aids na região de Ribeirão Preto, Estado de São Paulo, onde cento e noventa e oito (198) pacientes anti-HIV positivos foram examinados no período de julho de 1992 a agosto de $1994^{(18)}$, revelou que $61 \%$ apresentavam alterações oculares enquanto $39 \%$ não apresentavam nenhum tipo de alteração. Quanto às alterações oculares encontradas, os exsudatos algodonosos foram a manifestação mais comum e a retinopatia pelo CMV a infecção mais freqüente, seguida pela retinocoroidite por toxoplasmose. A maioria dos indivíduos (57,5\%) apresentava alguma doença sistêmica associada.

\subsection{Alterações da microvasculatura}

Constituem a chamada retinopatia da aids ou retinopatia pelo HIV, ou retinopatia não infecciosa pelo HIV. As alterações vasculares mais freqüentes são os exsudatos algodonosos, seguidos pelas hemorragias intra-retinianas e microaneurismas ${ }^{(19)}$.

A etiopatogenia de tais alterações não é totalmente conhecida, mas elas podem ser devidas a lesões do endotélio vascular pelo HIV $^{(20,21)}$; à deposição de imunocomplexos circulantes na parede vascu$\operatorname{lar}^{(22)}$ ou a alterações do fluxo sanguíneo retiniano. A diversidade dos achados sugere múltiplos fatores, infecciosos e não infecciosos. Ocorrem mais freqüentemente com baixa contagem de $\mathrm{CD}_{4}^{+{ }^{(15)}}$, geralmente, abaixo de 100 células $/ \mathrm{mm}^{3}$. O diagnóstico diferencial deve ser feito com a retinopatia incipiente pelo $\mathrm{CMV}$, a retinopatia pelo lúpus eritematoso sistêmico, pelo diabetes e pela hipertensão arterial. Tais exsudatos regridem espontaneamente em duas (2) a seis (6) semanas, sem deixar seqüelas. 


\subsection{Infecções Oportunistas}

As infecções oportunistas são as complicações oculares com maior impacto na função visual dos pacientes com aids ${ }^{(23,24,25)}$. Podem envolver o segmento anterior, o posterior e os anexos. Uma grande variedade de agentes patogênicos pode ser responsável pelas lesões oculares, como infecções virais (retinopatia pelo CMV e pelo Herpes zoster: lesões palpebrais pelo molusco contagioso), infecções bacterianas (coroidite pelo Mycoplasma e uveíte luética), infecções fúngicas (coroidite por Candida) e infecções por protozoários (ceratite por Microsporidium e retinite por Toxoplasma gondii).

\subsubsection{Retinopatia pelo Citomegalovírus}

A retinopatia pelo CMV é a maior causa de cegueira nos pacientes com aids e a causa mais freqüente das infecções oportunistas oculares na maioria dos estudos. Sua frequiência tem variado de 15 a $46 \%{ }^{(26)}$, acometendo pacientes que, via de regra, apresentam contagem de $\mathrm{CD} 4^{+}$abaixo de $100 / \mathrm{mm}^{3}(14,27)$.

Geralmente, o paciente apresenta poucos sintomas, dependendo da localização da lesão. Os mais comuns são: embaçamento visual, perda da visão central, escotomas, moscas volantes e fotopsia. Ao exame, observam-se, à oftalmoscopia, basicamente três formas de apresentação, isoladas ou sobrepostas: áreas brancas de necrose, com aparência granular, geralmente na periferia ou longe das arcadas vasculares; áreas de necrose com predominância de hemorragia e vasculite, proximamente às arcadas vasculares e vasculite intensa (galhos de árvore congela$\operatorname{dos})^{(23,26,28,29)}$. As áreas de necrose progridem lentamente, sendo a mácula acometida nos estágios mais avançados. A maioria dos pacientes apresenta um quadro unilateral no início, em 30 a $40 \%$ dos casos, e que pode se tornar bilateral em $60 \%$ ou mais dos pacientes não tratados ${ }^{(30)}$.

As drogas antivirais disponíveis na atualidade para o tratamento da retinopatia pelo CMV são o Ganciclovir, o Foscarnet e o Cidofovir ${ }^{(31,32)}$.

\subsubsection{Retinocoroidite por toxoplasmose}

É outra causa freqüente de retinite infecciosa na aids e, em alguns pacientes, é a manifestação inicial da doença ${ }^{(33)}$. Deve ser diferenciada da retinopatia pelo CMV com base nos aspectos clínicos e na resposta à terapêutica específica. As lesões são brancas e floconosas, sem o padrão granular da retinopatia pelo CMV e há maior reação vítrea do que nela.
Cerca da metade dos casos apresenta, concomitantemente, manifestações de acometimento neurológico (neurotoxoplasmose). Em contrapartida, os pacientes com doença neurológica pela toxoplasmose apresentam 10 a $20 \%$ de chance de apresentarem o quadro ocular. As medicações e dosagens utilizadas são as mesmas do tratamento utilizado nos imunocompetentes. Em adultos, a Sulfadiazina é empregada na dose de $1000 \mathrm{mg}$ via oral, de seis em seis (6/6) horas, e a Pirimetamina na dose de $50 \mathrm{mg}$, via oral, uma vez ao dia. O uso associado de corticosteróides é controverso nesses casos $^{(34)}$.

\subsubsection{Retinite herpética}

A necrose retiniana progressiva externa (PORN) e a necrose retiniana aguda (ARN) somam $1 \%$ das retinites infecciosas nos pacientes com aids ${ }^{(15)}$. A PORN é causada pelo vírus da varicela-zoster, e é caracterizada por necrose das camadas externas da retina, em áreas múltiplas do pólo posterior, que rapidamente coalescem e atingem todas as camadas retinianas. Há pouca hemorragia, e o vítreo é, geralmente, poupado. As lesões progridem rapidamente e de forma fulminante, a despeito da terapêutica ${ }^{(35)}$.

$O$ paciente queixa-se de perda da acuidade visual, sem dor. Ao exame oftalmoscópico, notam-se áreas de opacificação retiniana nas camadas profundas. O comprometimento vascular não é freqüente e o nervo óptico pode ser comprometido em $17 \%$ dos casos. O uso do Aciclovir, na dose de $20 \mathrm{mg} / \mathrm{Kg}$, a cada oito (8) horas, por via endovenosa, por duas (2) semanas, seguido pelo uso oral na dose de $800 \mathrm{mg}$, cinco (5) vezes ao dia, tem sido recomendado.

A ARN é outra forma de retinopatia causada pelo vírus Herpes zoster e pelo Herpes simplex ${ }^{(24,35)}$ e tem um prognóstico melhor que o da PORN. As lesões se iniciam na periferia e evoluem de maneira confluente e centrípeta, com maior reação inflamatória do vítreo e do segmento anterior, com sinais freqüentes de vasculite. A terapêutica pode ser feita com o Aciclovir por via endovenosa, na dose de $13 \mathrm{mg} / \mathrm{Kg}$, de oito em oito (8/8) horas, durante quinze (15) dias, seguido pelo uso oral, na dose de $800 \mathrm{mg}$, cinco (5) vezes ao dia.

\subsubsection{Retinite sifilítica}

As manifestações oculares da sífilis em pacientes com aids são mais severas, prolongadas, com maior chance de recorrência, e com maior comprometimento bilateral, quando comparadas com as que ocorrem nos pacientes imunocompetentes ${ }^{(36)}$. 
Além da coriorretinite, ela pode causar vasculite, uveíte e papilite. Há duas formas de retinite: um padrão branco-amarelado fora das arcadas vasculares ${ }^{(37)}$, e um padrão cinza esbranquiçado, de retinite profunda. O diagnóstico pode ser feito através de testes sorológicos como o FTA-Abs. Os pacientes são tratados com penicilina $\mathrm{G}$ cristalina por via endovenosa, na dose de dois (2) a quatro (4) milhões de unidades, a cada quatro (4) horas, durante catorze (14) dias consecutivos $^{(36,38)}$.

\subsubsection{Coroidite pelo Cryptococcus neoformans}

A ocorrência de infecção por fungos do gênero Cryptococcus é bastante comum em pacientes com aids ${ }^{(11,12)}$. A coroidite se apresenta como lesões múltiplas, amareladas. Esse quadro ocular não é freqüente, e, geralmente, se associa com a doença disseminada. As manifestações oculares mais freqüentes são inespecíficas e secundárias ao envolvimento do SNC. $\mathrm{O}$ tratamento pode ser feito com a Anfotericina-B ou com o Fluconazol ${ }^{(12)}$.

\subsubsection{Coroidite pelo Pneumocystis carinii}

Quando não recebem tratamento profilático adequado, aproximadamente $60 \%$ dos pacientes com aids estão sob risco de desenvolverem pneumonia pelo Pneumocystis carinii. Desses, menos de 1\% desenvolvem foco extrapulmonar, como a retina. A lesão retiniana é caracterizada por lesões múltiplas, bilaterais, amareladas, que podem coalescer e apresentam pouca reação inflamatória ${ }^{(39)}$. O tratamento pode ser feito com a associação de Sulfametoxal e Trimetoprim ${ }^{(12)}$.

\subsubsection{Infecções Oculares Externas}

A infecção mais comum no segmento anterior é o Herpes zoster oftálmico ${ }^{(40)}$. Os pacientes apresentam lesões de pele vesiculobolhosas no território do ramo oftálmico do trigêmeo e, além disso, podem apresentar ceratite e/ou uveíte. O quadro clínico costuma ser típico e o diagnóstico feito pelo aspecto das lesões. A terapia pode ser feita com Aciclovir por via endovenosa e/ou oral.

\subsection{Neoplasias}

As principais neoplasias associadas com a aids são o sarcoma de Kaposi e o linfoma ${ }^{15,20,24}$. O sarcoma de Kaposi é uma neoplasia vascular multicêntrica da pele, mucosas, órgãos internos e linfonodos. Acomete as pálpebras, a conjuntiva bulbar ou palpebral e a órbita, em cerca de $20 \%$ dos $\operatorname{casos}^{(41)}$. Clinicamente se apresenta como uma lesão violácea na conjuntiva, mais freqüentemente no fórnix inferior, que pode cur- sar com irritação, hemorragias subconjuntivais, infecção, triquíase e entrópio.

A quimioterapia e a imunoterapia são necessárias para controlar a disseminação do tumor, mas seu uso deve ser criterioso pois favorece as infecções oportunistas. A crioterapia e a retirada cirúrgica estão indicadas nas lesões isoladas ${ }^{(41)}$.

Os linfomas que acometem os pacientes com aids são do tipo não-Hodgkin , e podem ser classificados em linfomas primários do olho e do sistema nervoso central (SNC) e linfomas sistêmicos ${ }^{(42)}$. O acometimento do globo ocular no linfoma do SNC é comum. O tumor é multicêntrico, e o acometimento bilateral, freqüente. Geralmente, ocorrem em uma etapa mais tardia da infecção pelo HIV e o diagnóstico se baseia na demonstração de células neoplásicas no vítreo ou em lesões coriorretinianas ${ }^{(43)}$.

\subsection{Distúrbios Neurooftalmológicos}

São decorrentes do acometimento do sistema nervoso central pelo próprio HIV, por infecções oportunistas ou neoplasias ${ }^{(15,20,24)}$. O próprio HIV é o responsável pelo mais freqüente acometimento do SNC na aids, caracterizado por uma demência progressiva (complexo aids-demência). O quadro ocular secundário a esse distúrbio neurológico pode se caracterizar por alterações visuais, paralisias do olhar e perturbações do campo visual.

A meningoencefalite criptocócica é também muito freqüente, e a manifestação ocular mais comum nessa doença é o papiledema. O paciente pode apresentar ainda, como complicação do acometimento do SNC, paralisias do olhar, alterações pupilares, alterações do campo visual e amaurose. As mesmas alterações oculares podem ser observadas em pacientes com granulomas no SNC, secundários às infecções por toxoplasmose e tuberculose.

\subsection{Outras alterações}

São descritas também várias manifestações oculares nos pacientes com aids, inespecíficas. A presença de uma ceratoconjuntivite seca pode estar presente em $10 \%$ a $15 \%$ dos pacientes ${ }^{(44)}$. Alguns estudos têm evidenciado alterações do epitélio conjuntival dos pacientes, decorrentes de deficiências de vitamina A, casos em que há uma redução no número das células caliciformes e das células epiteliais da conjuntiva ${ }^{(45)}$. Defeitos da acomodação visual com dificuldades na leitura têm sido descritos e possivelmente estão associados com o estado geral, debilitado, do paciente ou com o uso de medicamentos ${ }^{(46)}$. 
FIGUEIREDO JFC et al. Some aspects of aids with interest to ophthalmologists. Medicina, Ribeirão Preto, 31: 577-583, oct./dec. 1998

ABSTRACT: Several medical specialties are currently facing the problem of aids in terms of diagnosis therapy, prophylaxis or investigation. Ophthalmologists play an important role in the diagnosis and therapeutic orientation of some clinical conditions of aids. The most common ocular complications in patients with aids are always due to exacerbation of latent infectious processes acquired before the immunosuppression. These infections tend to disseminate, causing varied systemic manifestations with clinical signs and symptoms that are not always characteristic. Ocular involvement is a mere local manifestation of dissemination of the process and ophthalmologic evaluation may be essential for establishment of diagnosis, since the alterations observed at opthalmoscopy may indicate the etiology of the process. In this respect, we review some aspects of aids that may be of interest to ophthalmologists.

UNITERMS: Ophthalmology. Eye Manifestations. Eye. Acquired Immunodeficiency Syndrome.

\section{REFERÊNCIAS BIBLIOGRÁFICAS}

1 - MASUR H; MICHELIS MA \& GREENE JB. An outbreak of community-acquired Pneumocystis carinii pneumonia: initial manifestation of cellular immune dysfunction. $\mathbf{N}$ Engl $\mathbf{J}$ Med 305:1432-1436,1981.

2 - GOTTLIEB MS et al. Pneumocystis carinii pneumonia and mucosal candidiasis in previously health homosexual men: evidence of a new acquired cellular immunodeficiency. $\mathbf{N}$ Engl J Med 305:1425-1431,1981.

3 - JOINT UNITED NATIONS PROGRAME ON HIV/AIDS (UNAIDS). Report on the global HIV/AIDS epidemic. World Health Organization, Geneva, June, 1998, 75p.

4 - MINISTÉRIO DA SAÚDE BRASIL. aids in Brasil. A joint government and society endeavour. XII World aids Conference, Geneva, June 28- July 3, 1998, 95p.

5 - PANTALEO G; GRAZIOLI C \& FAUCI,AS. The immunopathogenesis of human immunodeficiency virus infection. $\mathbf{N}$ Engl J Med 328:327-335, 1993.

6 - SHEPPARD HM. XI International Conference on AIDS. Berlim, June 6-11, 1993. Abstract WS-BO3-1.

7 - $\mathrm{CAO} Y$ et al. Virologic and immunologic characterization of long-term survivors of human immunodeficiency virus type 1 infection. N Engl J Med 332:201-208, 1995.

8 - GRAZIOSI-PANTALEO G et al. Kinetics of human immunodeficiency virus type 1 (HIV-1) DNA and RNA synthesis during primary HIV-1 infection. Proc Natl Acad Sci USA 90: 6405-6409, 1993.

9 - CENTERS FOR DISEASE CONTROL. 1993 Revised Classification System for HIV infection and expanded surveillance case definition for AIDS among adolescents and adults. MMWR 41: 1-13, 1992.

10 - MACHADO AA et al. Perfil clínico-epidemiológico de pacientes de Ribeirão Preto,São Paulo, Brasil, com AIDS e infecções oportunistas. MEDICINA, Ribeirão Preto 30:106-122, 1997.
11 - FIGUEIREDO JFC. Síndrome da Imunodeficiência Adquirida: aspectos sistêmicos. Reunião Jubileu de Prata do Centro de Estudos Oftalmológicos Cyro de Rezende. ANAIS, Legis Suma, Ribeirão Preto, p.125-130, 1989.

12 - FIGUEIREDO JFC \& MARTINEZ,R. Emergências em adultos portadores de Síndrome da Imunodeficiência Adquirida (AIDS). MEDICINA, Ribeirão Preto 25:505-513, 1992.

13 - HENDERLY DE et al. Cytomegalovirus retinitis as the initial manifestation of acquired immunodeficiency syndrome. Am J Ophthalmol 103:316-320, 1987.

14 - KUPPERMANN BD et al. Correlation betweem $\mathrm{CD}_{4}{ }^{+}$counts and prevalence of cytomegalovirus retinitis and human immunodeficiency virus-related noninfectious retinal vasculopathy in patients with acquired immunodeficiency syndrome. Am J Ophthalmol 115:575-582,1993.

15 - KREIGER AE \& HOLLAND GN. Ocular involvement in AIDS. Eye 2:496-505, 1988.

16 - MUCCIOLI C et al. Achados oftalmológicos em AIDS: avaliação de 445 casos atendidos em 1 ano. Rev Assoc Med Bras 40:155-158, 1994.

17 - RODRIGUES MLV; SOUZA NV \& FIGUEIREDO JFC. AIDS: Ocular manifestations in Brasil. Ribeirão Preto, FMRP-USP $13 p, 1996$.

18 - KRIEGER FTS. Estudo das manifestações oculares em portadores do vírus da imunodeficiência humana e em pacientes com a síndrome da imunodeficiência adquirida na região de Ribeirão Preto. Dissertação de Mestrado, Faculdade de Medicina de Ribeirão Preto da USP, p. 1 - 98, 1998.

19 - RAO K et al. Retinal microvascular abnormalities in the acquired immuno deficiency syndrome. Invest Ophthalmol Vis Sci 33:742, 1992. Abstract.

20 - POMERANTZ RJ et al. Infection of the retina by human immunodeficiency virus type 1. N Engl J Med 317:16431647, 1987.

21 - FABER DH et al. Role of HIV and CMV in the pathogenesis of retinitis and retinal vasculopathy in AIDS patients. Invest Ophthalmol Vis Sci 33:2345-2353, 1992. 
22 - PEPOSE JS et al. Acquired immunodeficiency syndrome. Pathogenetic mechanisms of ocular disease. Ophthalomology 92:472-484, 1983.

23 - PALESTINE AG et al. Ophthalmic involvement in acquired immunodeficiency syndrome. Ophthalmology 91:10921099, 1984.

24 - DAMICO DJ. Diseases of the retina. N Engl J Med 331:95106, 1994.

25 - HOLLAND GN. Acquired immunodeficiency syndrome and ophthalmology: the first decade. Am J Ophthalmol 114: 86-95, 1992.

26 - FREEMAN WR et al. A prospective study of ophthalmologic findings in the acquired immunodeficiency syndrome. Am J Ophthalmol 97:133-142, 1984.

27 - SPAIDE RF; GAISSINGER A \& PODHORZER JR. Risk factors for cotton-wool spots and cytomegalovirus retinitis in patients with human immunodeficiency virus infection. Ophthalmology 102:1860-1864, 1995.

28 - HOLLAND GN et al. AIDS: Ocular manifestations. Ophthalmology 90: 859-873, 1983.

29 - EGBERT PR et al. Cytomegalovirus retinitis in immunosupressed hosts. II-Ocular manifestations. Ann Intern Med 93:664-670, 1980.

30 - JABS DA; ENGER C \& BARTLETT JG. Cytomegalovirus retinitis and acquired immunodeficiency syndrome. Arch Ophthalmol 109:794-799, 1989.

31 - STUDIES OF OCULAR COMPLICATIONS OF AIDS RESEARCH GROUP IN COLLABORATION WITH THEAIDS CLINICAL TRIAL GROUP. Combination foscarnet and ganciclovir therapy vs monotherapy for the relapsed cytomegalovirus retinitis in patients with AIDS. Arch Ophthalmol 114:23-33, 1994.

32 - SHIGETA S et al. Comparative inhibitory effects of nucleoside analogues on different clinical isolates of human cytomegalovirus in vitro. J Infect Dis 163:270-275, 1991.

33 - WEISS A et al. Toxoplasmic retinochoroiditis as an initial manifestation of acquired immunodeficiency syndrome. Am J Ophthalmol 101:248-249, 1985.

34 - COCHEREAU-MASSIN I et al. Ocular toxoplasmosis in human immunodeficiency virus-infected patients. Am J Ophthalmol 114:130-135, 1992.
35 - ENGSTROM RE et al. The progressive outer retinal necrosis syndrome. A variant of necrotizing herpetic retinopathy in patients with AIDS. Ophthalmology 101:1488-1522, 1994.

36 - PASSO MS \& ROSENBAUM JT. Ocular syphilis in patients with human immunodeficiency virus infection. Am J Ophthalmol 106:1-6, 1988.

37 - STOUMBS VD \& KLEIN ML. Syphilitic retinitis in a patient with acquired immunodeficiency syndrome related-complex. Am J Ophthalmol 103:103-104, 1987.

38 - SHALABY IA et al. Syphilitic uveitis in human immunodeficiency virus-infected patients. Arch Ophthalmol 115:469473, 1997.

39 - RAO NA et al. A clinical, histophatologic and electron microscopic study of Pneumocystis carinii choroiditis. Am J Ophthalmol 107:218-222, 1989.

40 - COLE EL et al. Herpes zoster ophthalmicus and acquired immunodeficiency syndrome. Arch Ophthalmol 102:10271029, 1984.

41 - DUGEL PU et al. Treatment of adnexial Kaposi's sarcoma in acquired immunodeficiency syndrome. Ophthalmology 99:1127-1132,1992.

42 - FONT RL; LAUCIRICA R \& PTRINELY JR. Immunoblastic B-cell lymphoma involving the orbit and maxicillary sinus in a patient with acquired immunodeficiency syndrome. Ophthalmology 100:966-970, 1993.

43 - SIGEL MJ et al. Ten-year experience with primary ocular reticulum cell sarcoma (large-cell non-Hodgkim's lymphoma). Br J Ophthalmol 73:343-346, 1989.

44 - KADHEM M; KALISH SB \& GOLDSMITH J. Ophthalmologic findings in acquired immunodeficiency syndrome. Arch Ophthalmol 102:201-206, 1984.

45 - FIGUEIREDO JFC et al. Conditions of the ocular surface in patients with Acquired Immunodeficiency Syndrome. Exp Eye Res 59:S-112, 1994.

46 - NEWSOME DA. Non-infectious ocular complications of acquired immunodeficiency syndrome. Int Ophtalmol Clinic 29:95-97, 1989.

Recebido para publicação em 11/03/97

Aprovado para publicação em 08/12/98 